\title{
La Bibliothèque nordique, territoire boréal à la bibliothèque Sainte-Geneviève
}

Fonds relatif aux pays de langues scandinaves et finno-ougriennes le plus important en dehors des pays nordiques, la Bibliothèque nordique compte près de 190.000 documents à ce jour, dont plus de 106.000 unica dans le Sudoc. La collection s'est enrichie en 2017 d'un don d'ouvrages en lituanien, ouvrant davantage le fonds spécialisé de la bibliothèque Sainte-Geneviève sur les pays baltes, déjà représentés par l'Estonie depuis 1986.

La Bibliothèque nordique avait fait l'objet en 2001 d'un article très complet dans

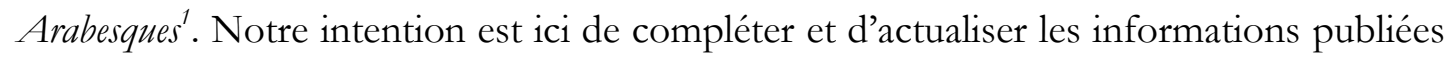
alors.

\section{Les 150 ans d'un don fondateur}

Forte d'une histoire de 150 ans, la collection fenno-scandinave de la bibliothèque Sainte-Geneviève fut créée en 1868 avec la réception du legs d'Alexandre Dezos de la Roquette. Consul de France à Elseneur au Danemark puis à Christiania (actuelle Oslo) en Norvège, Alexandre Dezos de la Roquette (1784-1868) fut également un bibliophile averti, vice-président et président honoraire de la Société de Géographie.

Ami de Ferdinand Denis, alors administrateur de la bibliothèque SainteGeneviève, Alexandre Dezos de la Roquette avait rassemblé une collection de 2.250 volumes imprimés et constitué un ensemble, numérisé en 2015 dans le cadre de BSN5, de 129 cartes marines et de 64 volumes d'archives consulaires et de manuscrits reliés.

Le don Dezos de la Roquette, outil précieux pour la connaissance des langues et cultures d'Europe du Nord, fut rapidement remarqué pour son caractère exceptionnel et les conservateurs et administrateurs exerçant à la bibliothèque Sainte-Geneviève lui adjoignirent des ouvrages issus du fonds ancien génovéfain, pour 500 d'entre eux issus du don Le Tellier (1710). Jean Mongin², Xavier Marmier ${ }^{3}$, Henri Lavoix ${ }^{4}$, Eugène Capet,

\footnotetext{
${ }^{1}$ N. Jullian et H. Vincenot. «La Bibliothèque Nordique », in Arabesques. Montpellier : Agence bibliographique de l'Enseignement Supérieur, janvier-février-mars 2001, p. 7- 9.

Qu'il soit rendu hommage ici à Madame Hedwige Vincenot, conservatrice en chef honoraire, qui dirigea la Bibliothèque nordique de 1984 à 2000.

${ }^{2}$ Auteur du Catalogue de la collection de livres scandinaves ou relatifs à la Scandinavie, cartes géographiques et manuscrits, donnée à la bibliothèque Sainte-Geneviève par M. Dezos de La Roquette, 1868. Cote : Ms 3641. Numérisé en 2015 et disponible en ligne à l'adresse : https://archive.org/details/MS3641.

${ }^{3}$ Xavier Marmier (1808-1892), fut administrateur de la bibliothèque Sainte-Geneviève, passeur des lettres scandinaves et germaniques, et membre de l'Académie française.

${ }^{4}$ LAVOIX, Henri. Rapport sur une mission en Suède, en Norvège et en Danemark, ayant pour but d'augmenter la Collection scandinave de la Bibliothèque Sainte-Geneviève, in Bulletin des Bibliothèques et des Archives, tome II ,1886, p.149-160 . Cote : 8 SC SUP 701 NOR.
} 
Erik Lie, Fritiof Palmér ${ }^{5}$, furent les premiers à contribuer à la description, au signalement et à l'enrichissement du don Dezos de la Roquette.

\section{Une bibliothèque de recherche ouverte à tous}

La Bibliothèque nordique, nommée ainsi en 1950 en raison de la pluralité des pays couverts (Danemark, Finlande, Iles Féroé, Islande, Norvège, Suède, puis une extension à deux pays baltes, l'Estonie en 1986 et la Lituanie en 2017) est le seul département de la bibliothèque Sainte-Geneviève à proposer le prêt à domicile, et ce, depuis la création de la première salle de lecture du fonds fenno-scandinave en 1903 au 8 place du Panthéon. Le prêt de 30 jours est possible pour la plupart des ouvrages parus après 1945, et ce service est apprécié des chercheurs scandinavistes comme du grand public, français ou étranger, qui peut s’inscrire gratuitement à la bibliothèque SainteGeneviève.

L'autre spécificité de la Bibliothèque nordique est la gratuité du prêt entre bibliothèques pour l'usager demandant des ouvrages conservés au Danemark, en Finlande, en Norvège et en Suède. Ce service permet de compléter une offre documentaire large dont l'accroissement dans toutes les sciences humaines est avant tout thématique. Ainsi, tous les ouvrages portant sur la Scandinavie sont acquis, de même que les traductions des langues nordiques au français. La Bibliothèque nordique offre à son public un ensemble allant des monographies anciennes aux publications académiques en anglais, en allemand, en français, la majeure partie de la collection étant en langue originale. Romans, ouvrages pour la jeunesse, beaux livres et bandes dessinées voisinent avec les ouvrages de la plus grande érudition sur les runes, sur Kierkegaard ou sur le modèle social scandinave.

\section{Au service du rayonnement et de la connaissance des pays nordiques}

Les échanges culturels et scientifiques entre les pays nordiques et la France sont nombreux et anciens, comme le montrent la présence de collèges scandinaves dans le Quartier latin dès l'époque médiévale ${ }^{6}$, ou encore la visite rendue à la bibliothèque de l'abbaye Sainte-Geneviève par le prince francophile qui devait devenir roi de Suède, Gustave III, en $1771^{7}$. Cet intérêt réciproque, régulièrement ravivé, entre les savants d'Europe du Nord et de l'Ouest, est sensible dans la bibliothèque particulière

\footnotetext{
${ }^{5}$ CAPET, Eugène, LIE, Erik et PALMÉR, Fritiof. Catalogue du Fonds scandinave de la Bibliothèque SainteGeneviève. XIII-[478] p. Chalon-sur-Saône,1908. Cote : JESSEN 63 NOR.

${ }^{6}$ Voir l'article de Guy de Valous dans La Bibliothèque nordique : Danemark. Finlande. Islande. Norvège. Suède. Lucie Thomas ; préface de Julien Cain ; Université de Paris, Bibliothèque Sainte-Geneviève. Paris: impr. Tournon, 1961. Cote : 8 SC SUP 89646 NOR.

${ }^{7}$ Deux visites royales à la bibliothèque de l'abbaye de Sainte-Geneviève (1764-1771) : notes de Mercier de SaintLéger publiée par Maurice Tourneux. Paris, 1897. Ex-dono : Maurice Tourneux à Henri Lavoix. Cote : BR. 69.243
} 
d'Alexandre Dezos de la Roquette, qui témoigne des relations nourries par ce donateur avec ses contemporains, savants et écrivains scandinaves ${ }^{8}$.

La richesse de la collection de la Bibliothèque nordique doit beaucoup à l'expertise des bibliothécaires étrangers, délégués par les pays scandinaves à la bibliothèque Sainte-Geneviève de la fin du XIXe siècle. jusqu'en 2005, la dernière bibliothécaire déléguée, Enrica Hallvarsson, exerçant actuellement à la Bibliothèque royale, c'est-à-dire nationale, de Suède. Les échanges se poursuivent à l'heure actuelle avec nos collègues d'Europe du Nord, qui contribuent à l'accroissement des collections en souscrivant des abonnements aux périodiques scientifiques les plus importants, et en envoyant régulièrement des ouvrages de Finlande, de Suède et de Norvège (ce dernier pays, par le biais de Biblioteksentralen, adresse plus de 300 ouvrages par an à la Bibliothèque nordique).

Les relations avec les Ambassades nordiques à Paris donnent également lieu à de nombreux partenariats (lancement d'ouvrages, soutien événementiel), et les nombreuses manifestations culturelles organisées par l'Institut suédois, par l'Institut finlandais, par la Maison du Danemark comme par les Ambassades de Norvège, d'Islande et d'Estonie, sont relayées à l'attention de nos lecteurs sur la page Facebook de la Bibliothèque nordique.

Enfin, les relations avec les universités partenaires sont primordiales. Avec la Sorbonne tout d'abord, dont la chaire de littératures scandinaves créée en 1909 est indissociable du développement de la Bibliothèque nordique, mais également avec les universités de Caen, de Strasbourg de Lille, de Lyon, et de Québec notamment, qui donnent lieu à des échanges professionnels réguliers. La tradition initiée par Régis Boyer, qui disposait jadis à la Bibliothèque nordique d'un bureau pour recevoir ses étudiants, se perpétue à présent sous la forme de conférences et de lectures, ou encore de séminaires portant sur les collections (séminaire conduit par Sylvain Briens sur les fonds d'autographes Prozor et La Chesnais), échanges qui suscitent des dons d'archives scientifiques.

\section{Valorisation et enrichissement des collections patrimoniales}

La valorisation des collections va de pair avec l'enrichissement des collections patrimoniales. Aux acquisitions courantes dans le cadre des marchés s'ajoutent en effet à la Bibliothèque nordique des acquisitions d'éditions originales, d'ouvrages anciens, de manuscrits, d'estampes, de reliures de création, et de livres d'artistes lors de ventes publiques ou auprès de libraires spécialisés en France comme à l'étranger.

Ces acquisitions ont permis d'enrichir les ensembles consacrés au Groenland, comme aux avant-gardes artistiques, ce qui a donné lieu à deux grandes expositions dans l'ensemble de la bibliothèque Sainte-Geneviève : l'exposition Pays vert, terre de glace : le

\footnotetext{
${ }^{8}$ Citons notamment une belle dédicace adressée par le poète romantique norvégien Henrik Wergeland à M. de la Roquette au seuil de l'édition originale du poème Den engelske Lods : et digt. Cote : 8 LA ROQ 366 NOR.
} 
Groenland hier et aujourd'bui (2015-2016) et Livres d'artistes CoBrA (2017-2018), expositions accompagnées de cycles de conférences.

\section{CollEx et SGBm : les deux défis actuels}

Il n'existait jusqu'ici pas de CADIST consacré aux études nordiques en France. L'attribution du label CollEx pour les études nordiques à la Bibliothèque nordique au début de l'année, permet au réseau documentaire formé avec la BU Tove Jansson de Caen et la BNUS de bénéficier d'une plus grande visibilité. Avec le soutien du groupement d'intérêt scientifique (GIS) établi à Strasbourg, il s'agira de doter les laboratoires d'études nordiques d'une plateforme combinant les outils bibliographiques utiles à la recherche et la valorisation des publications francophones dans ce domaine. Le travail conjoint avec les bibliothécaires allemands à l'origine du portail ViFaNord permettra au réseau documentaire français de proposer la même ouverture sur l'Europe que l'Association Pour les Etudes Nordiques fondée en 2013 à Strasbourg par le réseau de chercheurs intéressés par nos collections.

Enfin, l'implication de la bibliothèque Sainte-Geneviève dans le projet de Système de gestion de bibliothèque mutualisé (SGBm) porté par l'ABES donne à la Bibliothèque nordique l'occasion de mettre à jour les notices présentes dans le seul SIGB local. Ce travail d'importance, visant à supprimer les scories afin que la migration de notices se passe au mieux, est un objectif prioritaire pour la fin de l'année 2018 à la Bibliothèque nordique comme dans le reste de l'établissement. Une opération de catalogage rétrospectif cofinancée par l'ABES a été entreprise au début de l'année 2018 afin de signaler dans le SUDOC les ouvrages qui avaient fait l'objet d'une description minimale dans le catalogue local, en particulier les ouvrages du fonds Tessin, issu du don de l'Institut suédois. Cette collection d'histoire de l'art, dont la numérisation a commencé en 2017, bénéficiera bientôt du rayonnement qu'elle mérite grâce à l'élan donné par le SGBm.

Florence CHAPUIS

Chef du département de la Bibliothèque nordique à la bibliothèque Sainte-Geneviève. 